\title{
Light and Velocity Variability in Proto-Planetary Nebulae
}

\section{Bruce J. Hrivnak and Wenxian Lu}

\section{Valparaiso University, Valparaiso}

We have been carrying out a program of monitoring light and velocity variability in protoplanetary nebulae (PPN). Variability might arise due to binarity or pulsation. Many planetary nebulae display a bipolar shape, and it is suggested that this is caused by binarity. This can be investigated in PPN, particularly by radial velocity studies of those of spectral types F and G. Pulsation is known to exist in other types of post-AGB stars, and can be a means to learn more about the physical properties of the stars. No previous study of a sample of PPN has been published.

Radial velocity studies have been carried out of 9 PPN over a 5 year period at the Dominion Astrophysical Observatory. They have been observed an average of 35 nights each, with a precision of $0.65 \mathrm{~km} \mathrm{~s}^{-1}$. All 9 are found to vary, with an average range of $10 \mathrm{~km}$ $\mathrm{s}^{-1}$. A period search was carried out using the CLEAN algorithm. We find 3 to display a clear period, ranging from 89 to $127 \mathrm{~d}$, but with varying amplitudes.

A light curve study has been initiated with the Valparaiso University $0.4 \mathrm{~m}$ telescope and CCD. In this expanded study, 40 PPN candidates with a full range of spectral types have been monitored for light variation. Several initial results have been found on the basis of the first 2 years of study. (1) Periodic variability was found in 9, with periods ranging from 25 to 140 days, although with varying amplitudes. These objects have spectral types F and G. (2) Short-time scale variability ( $<10 \mathrm{~d}$ ) was found in many of the objects, especially those of early spectral types, $O$ and B. (3) Several have unusual light curves, such as the appearance of a periodic variation in one season and not the next, or of an unusual shape to the light curve. This light curve monitoring program is continuing.

Several preliminary conclusions can be drawn on the basis of this variability study.

1. Almost all PPN vary in light and velocity.

2. For the 3 cases with periodic velocity variability, the light varied with the same period; however, the phase relationship between the 2 curves was different in each case.

3. The variability is due to pulsation; no binaries were discovered.

4. The variability does not have a simply periodic form; instead there exists varying amplitudes, varying periods, or multiple periods.

5. There exists a general trend such that those with longer periods are those with later spectral types. 